\title{
Papás Saludables, Niños Saludables: Perspectives From Hispanic Parents and Children in a Culturally Adapted Father-Focused Obesity Program
}

\author{
Oriana Perez, $\mathrm{MS}^{1}$; Alicia Beltran, MS, RD ${ }^{1}$; Tasia Isbell, $\mathrm{MPH}^{2}$;
}

Edgar Galdamez-Calderon, $\mathrm{BA}^{1}$; Tom Baranowski, $\mathrm{PhD}^{1}$; Philip J. Morgan, $\mathrm{PhD}^{3}$; Teresia M. O'Connor, MD, $\mathrm{MPH}^{4}$

\begin{abstract}
Objective: Qualitatively assess culturally adapted lifestyle intervention, Papás Saludables, Niños Saludables (PSNS; Healthy Dads, Healthy Kids), for Hispanic fathers and children.

Methods: Semistructured interviews of parents and children after participation in 10-week PSNS program for Hispanic fathers and children. Qualitative data double-coded inductively and deductively until consensus reached.

Results: Total of 26 fathers, 26 mothers, and 45 children interviewed. Parents and children had positive feedback about program content on culturally relevant nutrition and physical activity and reported improved father-child bonding. Mothers noted increased involvement among fathers in child's well-being. Participants suggested lengthening the program.

Conclusions and Implications: Papás Saludables, Niños Saludables is an innovative approach with promise in engaging Hispanic fathers and children in a lifestyle program that emphasizes the role of fathers in children's lifestyle behaviors. Familism, respeto (respect), and promoting father-child relationships were important to engage fathers. Results from this study will inform future trials of PSNS and help identify ways to increase engagement of Hispanic men in other programs.
\end{abstract}

Key Words: Hispanic, fathers, children, obesity prevention, culturally adapted (J Nutr Educ Behav. 2021;53:246-253.)

Accepted November 14, 2020. Published online December 22, 2020.

\section{INTRODUCTION}

Fathers have an important role in children's nutrition, physical activity, and weight. ${ }^{1-3}$ However, most obesity prevention programs have focused on mothers. ${ }^{2,4}$ There are even fewer programs focusing on Hispanic fathers. ${ }^{5}$ A longitudinal study in Australia identified that children with an obese father were more likely to become obese than children whose father had a healthy weight, whereas having an obese mother did

\footnotetext{
${ }^{1}$ Department of Pediatrics, US Department of Agriculture/Agricultural Research Service Children's Nutrition Research Center, Baylor College of Medicine, Houston, TX

${ }^{2}$ School of Public Health and McGovern Medical School, The University of Texas Health Science Center at Houston, Houston, TX

${ }^{3}$ Priority Research Centre in Physical Activity and Nutrition, Faculty of Education and Arts, University of Newcastle, Newcastle, New South Wales, Australia

${ }^{4}$ Department of Pediatrics, US Department of Agriculture/Agricultural Research Service Children's Nutrition Research Center, and Academic General Pediatrics, Baylor College of Medicine, Houston, TX

Conflict of Interest Disclosure: The authors have not stated any conflicts of interest.

Address for correspondence: Teresia M. O'Connor, MD, MPH, Department of Pediatrics, USDA/Agricultural Research Service Children's Nutrition Research Center, and Academic General Pediatrics, Baylor College of Medicine, 1100 Bates St, Houston, TX 77030; E-mail: teresiao@bcm.edu

(C) 2020 The Authors. Published by Elsevier Inc. on behalf of Society for Nutrition Education and Behavior. This is an open access article under the CC BY-NC-ND license (http:// creativecommons.org/licenses/by-nc-nd/4.0/)

https://doi.org/10.1016/j.jneb.2020.11.006
}

not appear to have the same effect on children. ${ }^{1}$ This was corroborated in other countries. ${ }^{6-8}$ The unique influence of fathers on their children's obesity-related behaviors ${ }^{9}$ makes them an important intervention target. Not engaging Hispanic fathers in obesity prevention is therefore concerning because Hispanic children in the US have one of the highest rates of obesity compared with children of other ethnic groups, with $46.2 \%$ of Hispanic children aged 6-11 years being overweight or obese. ${ }^{10}$ Hispanic men also have higher rates of obesity and overweight than nonHispanic white individuals. ${ }^{11}$ Hispanic men are often thought of as a hard-to-reach population, especially for weight loss programs. ${ }^{12,13}$ However, they merit more attention as fathers' roles have evolved over the last several decades. ${ }^{14}$

Papás Saludables, Niños Saludables (PSNS) is a healthy lifestyle program for fathers that was culturally adapted from Healthy Dads Healthy Kids 
(HDHK), ${ }^{15,16}$ a program initiated in Australia. Based on Social Cognitive Theory, ${ }^{17}$ PSNS engages fathers and children together to promote healthy eating, physical activity for weight loss for fathers, and obesity prevention for children aged 5-11 years. The program stresses the importance of fathers in their children's lives by addressing self-efficacy, role modeling, and social support among fathers and their children. In addition, PSNS is based on Family Systems Theory, in which 1 family member can affect the behavior of the other family members through reciprocal reinforcement. ${ }^{15,18,19}$ The original program, HDHK, was evaluated and implemented in efficacy, ${ }^{15}$ effectiveness, ${ }^{16,20}$ and dissemination trials ${ }^{21}$ in Australia. Results from these trials showed that HDHK had a significant impact on fathers' weight loss and on positive lifestyle behavior changes for fathers and children.

Papás Saludables, Niños Saludables was culturally adapted from its original Australian version to better respond to the cultural values and context of Hispanic families living in the US. ${ }^{22}$ The adaptation was based on the Ecological Validity Model, with the aid of a panel of Hispanic families and an expert panel of researchers. ${ }^{22}$ Cultural values such as familismo (familism), respeto (respect), and colectivismo (collectivism) ${ }^{23}$ were emphasized. The program material was changed to reflect US recommendations for physical activity, ${ }^{24}$ dietary intake, ${ }^{25}$ screen media use for men and children, ${ }^{26}$ and common food and games among Hispanic families in this region. ${ }^{22} \mathrm{~A}$ booster session was added at the midpoint of the program to review the previously learned content and clarify areas of misunderstanding on important topics.

The resulting 10-week group-based PSNS program was assessed for feasibility among a sample of Hispanic families in a randomized, wait-list controlled trial. The feasibility findings have been published. ${ }^{27}$ Understanding the participant's experience in the feasibility assessment of a program is important to inform the next steps for the program. The Practical, Robust Implementation and Sustainability Model (PRISM) $^{28}$ provides a path to translate research into practice when a program is feasible, efficacious, and effective in research studies. $^{28,29}$ The Practical, Robust Implementation and Sustainability Model considers the external environment, the infrastructure to implement and sustain the program, and the perspectives of the program recipients. In this study, the patient perspective constructs of the PRISM model were applied post hoc to describe Hispanic fathers', mothers', and children's experiences in the program to better understand how family members engaged with PSNS. A secondary aim was to identify ways to improve and enhance PSNS for future clinical trials. This article, therefore, presents the qualitative perspectives of fathers, mothers, and children gathered after program participation.

\section{METHODS}

A total of 36 families (36 fathers, 35 mothers, and 64 children) were recruited to participate in the PSNS feasibility trial during fall 2018 and spring 2019 (Clinicaltrials.gov registration number NCT03532048). Families were recruited by research staff in the participating clinic's waiting room and by fliers posted in the clinic. A total of 19 families were randomized to the intervention group and 17 to the wait-list control group. Both groups participated in the program approximately 5 months apart. Inclusion criteria were being a father or a father figure of a Latino or Hispanic origin (ie, stepfather, adult brother, grandfather, uncle, etc) of a child aged 5-11 years who was a primary care patient at the Center for Children and Women clinic (part of the Texas Children's Health Plan, a Medicaid and Children's Health Insurance Plan provider), able to speak and read English or Spanish, having a body mass index between 25 and $40 \mathrm{~kg} / \mathrm{m}^{2}$, and passing a health screening to engage in exercise. ${ }^{30} \mathrm{Up}$ to 3 children per family were enrolled.

All families who could be reached after the program concluded were asked to take part in an exit interview. Of these, 26 fathers, 26 mothers, and 45 children agreed to participate in the exit interview. All participants provided informed consent and assent for children. The Institutional Review Board of Baylor
College of Medicine reviewed and approved this study.

Papás Saludables, Niños Saludables was previously described in detail ${ }^{27}$ and is only briefly described here. The 10-week, group-based program consisted of 90-minute sessions, including a 10-15 minute warm-up with fathers and children together, separate 30-minute breakouts for fathers (Dad's Club) and children (Kid's Club), followed by 45 minutes of physical activity with fathers and children (Sports Club). The program was delivered in English and Spanish by 3 bilingual facilitators.

The Dad's Club sessions included topics such as the importance of fathers in their child's health, how to lose weight in a healthy and sustainable way, nutrition, reducing screen time, increasing physical activity, and parenting. The children had sessions corresponding to the topics covered for fathers. The Sports Club consisted of luchitas (play wrestling or rough-and-tumble play), fundamental movement skills (sports skills), and fitness exercises. The fathers and their children attended all sessions together and received a handbook with the information presented, as well as home tasks to be completed during the week. Families were provided with a pedometer and sports equipment to practice sports skills at home. Mothers were invited to participate in 1 session, received a handbook and weekly videos, and were invited to a private Facebook (Facebook, Inc, Menlo Park, CA) group.

\section{Qualitative Data Collection}

Participating fathers, mothers, and children were interviewed shortly after the conclusion of each wave of the PSNS program. Interviews for the intervention group took place during December, 2018 and during April, 2019 for the wait-list control group. The interviews were conducted inperson or over the telephone in English or Spanish by either of 2 bilingual staff with graduate-level and inhouse training and experience in qualitative research. Interviewers used a standardized script, which was adapted from the original Australian HDHK program. ${ }^{15}$ It included openended questions, probes, and prompts 
to clarify responses. The interviews were audio-recorded, transcribed verbatim professionally, and translated into English if necessary.

The interviews for fathers and mothers explored (1) motivations and reasons for participating in the program, (2) experiences participating in the program, (3) impact of the program on their lives and families, and (4) suggestions for program improvement. In addition, the interviews explored the mothers' experiences with the content delivered to them through Facebook posts and online videos (interview scripts available on request). Families received compensation for completing the data collection but not for attending the program sessions. Fathers and mothers each received compensation of \$30 for completing the interviews.

Owing to the young age of some of the children, their interviews were semiquantitative to better capture their thoughts. Children were interviewed by a research staff member, and for some questions, they were provided a list of response options, whereas other questions were openended. The interviews were audio-recorded, and notes were taken by the staff. The interviews explored (1) their favorite and least favorite parts about the program, (2) their perceptions about attending the program with their father, (3) helping their father become healthier, (4) impact of the program on their time spent with their father, and (5) suggestions for improvement. One interview recording was not retrievable and not included in this manuscript.

\section{Analysis}

The English interview transcripts were double-coded thematically and independently by pairs of research staff trained in qualitative analysis, using NVivo (version 11, QSR International, Burlington, MA, 2015). The patient perspective dimension of PRISM $^{28}$ served as a theoretical basis to organize the analysis post hoc. The patient perspective dimension includes (1) patient centeredness, (2) patient choices, (3) patient barriers, (4) seamlessness of transition between program elements, (5) services and access, (6) burden (complexity and cost), and (7) feedback of results. In the context of the PSNS program, the focus was on participants instead of patients. Separate codebooks were created for the fathers', mothers', and children's interviews. The codebooks included inductive and deductive themes. Secondarily, themes were organized according to the PRISM model dimensions. The fathers', mothers', and children's data were each analyzed by pairs of primary coders and 1 external reviewer, with a total of 5 coders across all the interviews. Differences in coding were discussed and reviewed among the coders and the external reviewer until a consensus was reached. The organization of themes into the PRISM dimensions was done by the 2 lead coders and reviewed with the principal investigator. The interviews of 10 children contained limited answers, mainly because of their young age, and did not contribute much to the results.

\section{RESULTS}

The fathers' ages ranged from 26 to 46 years (median, 36 years), and their mean BMI was 28.6 (SD, 2.55) $\mathrm{kg} / \mathrm{m}^{2}$. The children's ages ranged from 5 to 11 years (median, 7 years), and 38\% were overweight or obese. Most of the fathers were born outside the US. Mexico was the most common country of origin, followed by El Salvador, Honduras, or other. Most of the fathers $(83 \%)$ stated they spoke only Spanish at home. The fathers tended to have a low educational status, with approximately two thirds having completed only some high school or less. Most (92\%) reported they had a family income of less than $\$ 47,000$ a year. Across the 2 groups, the 28 fathers who started the program on average attended $72 \%$ of the 10 PSNS sessions, ${ }^{27}$ whereas the 26 fathers who took part in the exit interviews on average attended 76\% of the sessions.

\section{Participant Centeredness}

Participant centeredness relates to whether the program addressed the values, preferences, and needs of the target population. The fathers stated their motivations to join PSNS included wanting to spend more time and improve the bond with their children, improve their health, exchange ideas with other fathers, curiosity, and because their spouse or partner encouraged them. The mothers decided to enlist their families in PSNS to encourage fathers and children to spend more time together and improve the father-child relationship and to learn more about nutrition and physical activity. The Table presents illustrative quotes from parents and children, corresponding to each dimension and categorized by themes.

A few mothers felt excluded and thought they should have been invited to attend all the sessions, highlighting that not all the mothers' preferences were met. Approximately half of the fathers reported they would have liked mothers to be more included in the program. They felt children would have liked it, and mothers would have benefited from the program. However, the other half thought it was good for the children to be away from their mothers, develop more independence, and interact more with fathers instead. Two fathers stated they would feel shy exercising around women.

\section{Participant Choices}

Before starting, the participants were offered to attend the PSNS on Saturday morning or Sunday afternoon. Only the Sunday afternoon time was selected, as this appeared to avoid potential conflicts with work for the fathers, who typically work 6-7 d/wk and have other family commitments. Fathers and mothers stated they would have liked the option to have more than 10 sessions or have longer than 90-minute sessions to complete the exercises and activities because the activities felt rushed, although recognizing not everyone would be able to attend.

Papás Saludables, Niños Saludables provided choices for in-home-based tasks. More than half of the fathers stated they were able to complete some of the home tasks such as walking 10,000 steps a day and trying a new fruit or vegetable with their children. The mothers were given the option to view weekly videos and engage in a Facebook group. 
Table. Representative Parents' and Children's Quotes for the Practical, Robust Implementation and Sustainability Model Dimensions

\section{Main Themes}

- Spend more time together

- Improve father-child relationship

- Learn more about nutrition and physical activity

- Spouse encouraged father to join

- Home-based tasks

- Schedule conflicts with program

- Home-based tasks

- Exercising outdoors

- Children encouraged their father to be healthier

- Luchitas at home

- Home-based tasks popular

- Facebook content for mothers

- Add more time to program

- Change program location

- Include mothers more

- Pedometer and work equipment barriers

\section{Participant centeredness}

"What I liked about the program the most, the games, the outdoor activities with the children. Sharing with my children." (father, wait-list control group $^{\mathrm{b}}$

"Because I think it is a good option ... to help them with the relationship between the dad and the kids as well as them working out together, how to eat..." (mother, intervention group)

"Well, my wife told me about it, I didn't know what it was, to be honest, but I knew that there were going to be activities for the children, and I liked the idea." (father, intervention group)

\section{Participant choices}

"The challenges that we did, we all had, to do maybe minimum half of an hour of exercise, or walking or to play with him (his child)."

(father, intervention group)

\section{Participant barriers}

"I did miss several [sessions], like four or five times I missed, I don't remember well. ...Because I had work, I had little jobs to finish...." (father, wait-list control group)

"In the cold weather, well, we were here at home, and during warm weather we went outside (to practice the sports skills)... In a small park that is here outside of the apartment." (father, intervention group)

"I made him put his phone down and spend more time playing with us ... He's putting [his phone] to the side more. ...Because he used to be on his phone all day." (child, wait-list control group)

\section{Seamless transition between program elements}

"Well, now-since the classes started, we play almost every night before going to bed, tickling, or luchitas ... they come and we play 15 or 20 minutes...." (father, wait-list control group)

"Oh, I liked it (the Facebook group) a lot, because they kept us up to date with what was happening and the topics that they were seeing. They also reminded us that the sessions were every Sunday. They reminded us of the hour, right? I also liked it, because they sent photos, and you could see the activities that they did every Sunday." (mother, wait-list control group)

\section{Service and access}

"Something that I didn't like was that the program was very short. I think it should be more consistent. More frequent, longer. The program was very fast. It seemed rushed. 'Our time is up now. We have to do this and this.' I didn't like that." (father, wait-list control group)

"If it could happen twice a week, and in a place that is more open, because we did have a couple accidents there with the children, for the same reason, because the parking lot is very hard." (father, wait-list control group)

"... for it (the program) to be healthy families, healthy kids. I mean, for it to also include the mom more also, if she wants to come and listen to the talks, or - I mean, for it to be more-to include everyone." (mother, intervention)

\section{Burden (complexity and cost)}

"I tried to use it (pedometer) but at work, since we are spraying and everything, we are in the dust and all that, it's not-it's sometimes also cumbersome because you get stuck with something." (father, wait-list control group) 
$250 \quad$ Perez et al

- MyFitnessPal (MyFitnessPal, Inc, San Francisco, CA) usage barriers

- Spend more time together

- Improve father-child relationship

- Spend more time with dad

- Learn about healthy eating

- Weight loss during program
Journal of Nutrition Education and Behavior Volume 53, Number 3, 2021

"I didn't use it, I did download it and I had it there, but I had issues with entering the application, and I stopped. I didn't use it." (father, intervention group)

\section{Feedback of results}

"...the program made me see where I was neglecting the area of the family, the children... .because one gets so immersed in work..." (father, intervention group)

"What I liked the most about everything was that the dads could have more of a relationship with the kids." (mother, intervention group)

"They (fathers) get involved more and they don't leave everything to us (mothers), because they know that the children also need time with the dad, and I think he learned that, being there in the program." (mother, wait-list control group)

"Well, I liked it, [be]cause, like I mentioned before, so I can have more time spending with my Dad. Also, be more healthy than before." (child, intervention group)

"To eat healthier, what potions, what type of portions to eat, if I am going to eat a plate, not to eat another one. . . sometimes even if I am full, I keep eating and it doesn't have to be like that...." (father, wait-list control group)

"My goal was to lose weight and I didn't achieve that, but I did accomplish many other things which were being closer to my children, spending more time with them, so I believe it was more valuable." (father, wait-list control group)

${ }^{a}$ Fathers $(n=26)$, mothers $(n=26)$, and children $(n=45) ;{ }^{b}$ Respondent, group in which they participated.

Note: The quotes represent the main themes organized by the Practical, Robust Implementation and Sustainability Model patient perspective dimension. ${ }^{28}$ Not all themes include a quote. No 1 participant provided more than 2 representative quotes to illustrate the breadth of comments obtained from the exit interviews.

Generally, the mothers found these useful but not all engaged with the options.

\section{Participant Barriers}

Family commitments and minor injuries from work were the most common reasons that prevented the fathers from being able to attend all 10 PSNS sessions and complete the home-based tasks. Program reminders were sent through text message, phone calls, voice mail, and weekly Facebook posts with pictures to remind the participants to attend the next session. The participants stated they appreciated the Facebook post reminders.

Owing to concerns about unsafe neighborhoods and lack of public parks as an impediment to outdoor activities for some families, indoor games and exercises were offered as an option. The fathers described that they were able to use the program games and the equipment at home or at local parks to continue practicing the activities with their children.

One of the core components of the program was to teach children that they could help encourage their fathers to eat healthier, be more physically active, and reduce their screen time. The fathers were informed that this was a program component, and children were encouraged to do it in a respectful way. Most children mentioned they were able to encourage their fathers to be healthier. Four children mentioned that their fathers had a negative reaction to their efforts, by not listening, getting mad, or asking them to stop trying.

\section{Seamless Transition Between Program Elements}

The fathers were encouraged to continue practicing the program activities at home. Luchitas and health challenges were the most popular activities that the fathers and the children routinely practiced at home. The mothers received the program content through videos and Facebook posts. They reported that this encouraged them to support what their spouses/partners and children learned during PSNS.

\section{Service and Access}

The program was offered in 2 classrooms and the parking lot of the clinic where the participating children usually receive their medical care. Suggestions from the fathers included offering the program at a park or other grassy area to have more space and prevent potential injuries while exercising on the pavement, more advanced nutrition content on diabetes and cholesterol, and more challenging exercises. Approximately half of the mothers expressed that they would like to have been more included in some way during the program. Many mothers had problems viewing the weekly videos owing to poor Internet access or other technical issues with their mobile devices.

\section{Burden (Complexity and Cost)}

Papás Saludables, Niños Saludables was provided free of cost. Transportation was available, but it was never requested or brought up by the participants as a barrier. Tracking food intake on MyFitnessPal (MyFitnessPal, 
Inc, San Francisco, CA) was burdensome for most fathers owing to a lack of time or to technical difficulties with using the phone application. Pedometers were used primarily for tracking during the first week they were distributed. Using the pedometer for the duration of the program was a burden for some fathers because it got in the way of work equipment.

\section{Feedback of Results}

Fathers, mothers, and children found that the program allowed fathers and children to spend more time together. The fathers reported that the program helped them improve their connection and communication with their children. They learned how to interact more with their children by using the games and the exercises they learned in PSNS as the means. The mothers liked seeing the improved relationship between the fathers and the children and that they spent more time together.

Not all of the fathers were able to reach their goal of maintaining or losing weight or identified weight loss as an important outcome for them. However, the fathers reported that they enjoyed learning about healthier versions of typical Hispanic and regional food, as well as learning to read food labels and appropriate portion sizes. Parents reported they implemented changes in family dietary habits, such as eating more fruits and vegetables and eating out less. In addition, the mothers indicated that the fathers were more involved in meal preparation, grocery shopping, and other chores.

A list of options was given to the children to choose their favorite and least favorite parts about the program (see Supplementary Data for all rankings). The ability to spend more time with their fathers was commonly mentioned by children as their favorite part of the program. Although the handbook was the least favorite because it felt like homework, there was not always enough time to finish it, or it was too difficult for the younger children and too easy for the older children.

The fathers suggested that the program should add more advanced nutrition content and encourage more discussions among fathers during Dad's Club. The mothers indicated that they would have liked to participate more in the program, either in the exercises, cooking classes, or other activities.

\section{DISCUSSION}

This study was undertaken to report the perspectives of Hispanic parents and children after participating in PSNS, organized through the patient perspective dimension of the PRISM framework. Few weight loss or childhood obesity prevention studies have targeted Hispanic fathers. ${ }^{5}$ It is therefore especially important to obtain the perspectives of this understudied population to understand how to best engage and retain Hispanic fathers and families in similar programs.

Many of the fathers in PSNS worked in physically demanding jobs $^{27}$; fatigue and minor work-related injuries were a concern for some. Other studies have found that Hispanic men in physically demanding jobs struggle with the need to engage in leisuretime physical activity. ${ }^{12,31,32}$ However, for many fathers in PSNS, the luchitas and the physically active games, accompanied by the prospect of an improved relationship with their children, were some of the most important aspects of the program that attracted them. This speaks to the importance of familismo when engaging Hispanic men. ${ }^{23}$ Even though some fathers and mothers said they would have liked the mothers to participate more to include the whole family, approximately half of the fathers raised concerns that including the mothers more would take the focus away from the fathers, which is what makes HDHK and PSNS unique. ${ }^{15,16,20}$ In addition, this may result in fathers delegating the responsibility of the program and its content to the mothers. Although familismo was an important theme of these exit interviews, it was nuanced to underscore the importance of focusing on fathers in the program.

The nutrition content was relevant and enjoyable to the fathers, mothers, and children who took part in PSNS. A recent review found that Hispanic mothers were more likely to engage in parenting around child feeding, whereas fathers get more involved in children's screen use and physical activity and sports on the basis of qualitative data. ${ }^{5}$ A diabetes prevention study for Hispanic families attended primarily by mothers reported one of the main barriers to changing the nutrition habits of the family was their husbands and their grandparents. ${ }^{33}$ In contrast, PSNS fathers and mothers reported that the fathers were working on increasing their fruit and vegetable intake and prepared more healthy meals with their children. Quantitative studies support that Hispanic fathers influence their children's eating, ${ }^{5}$ suggesting these changes can affect both the fathers' and children's nutrition behaviors. By contrast, the weight loss aspect of PSNS was not as relevant for all the fathers. Some fathers were successful in losing or maintaining weight. However, for some fathers, this was secondary to their goal of spending more time with their children. Studies looking at weight loss program interventions for Hispanic populations have shown that those that include spouses and children have better outcomes than those that focus on the individual, ${ }^{32}$ suggesting that the weight loss component may still be relevant for some.

A component that raised some concern before implementing the program $^{22}$ was the reaction of the fathers to their child's encouragement to be more physically active and eat healthier. However, only 4 children reported having difficulties with this element of the program. It is possible that the emphasis on the value of respeto for children during the program may have helped mitigate this issue. This finding may be important for other programs that focus on Hispanic families.

The exit interviews identified several areas to consider for improvement in future versions. The child handbook Kid's Club activities should be reviewed and made to feel less like school work. Better locations with access to fields should be considered for the outdoor Sports Club delivery. Additional, optional time can be 
added to the end of the Sports Club for those who desire to stay longer and keep practicing the skills and the exercises. The technical difficulties for some mothers in watching the weekly videos need to be investigated and addressed.

This study had several limitations. The Hispanic population in the US is a heterogeneous group by country of origin, immigration and acculturation status, income, and educational levels. ${ }^{34}$ The results may not be generalizable to other Hispanic families from a different background than those recruited in this study, who were low income, qualified for Medicaid, and primarily from Mexico and Central America. The interview guide was not developed to address the 7 concepts of the patient perspective for PRISM; instead, the framework was used post hoc to organize the findings. In addition, this study included the viewpoints from individuals who had participated in the program. It did not include feedback from those fathers and families who enrolled in the study but did not start the PSNS program. Understanding barriers and motivations for not participating will be important in the future. In addition, there was the potential for social desirability bias toward the interviewer.

\section{IMPLICATIONS FOR RESEARCH AND PRACTICE}

The father-targeted PSNS program was a promising way to get Hispanic fathers engaged and change their health-associated behaviors. A 10week, participant-centered innovative intervention targeting Hispanic fathers to promote a healthy lifestyle for themselves and their children was well received by fathers, mothers, and their children. The program offered choices in how to engage participants in between sessions, with a strong focus on familism and building father-child relationships. Fun physical activities that fathers and children can complete together and culturally relevant nutrition education are promising strategies. The emphasis on cultural values such as familismo and respeto emerged as important when engaging Hispanic fathers. The
PSNS program appears to have strong potential to engage Hispanic fathers, improve child and father relationships, and improve health behaviors among Hispanic families. Future trials need to test the efficacy, reach, and sustainability of PSNS among Hispanic families.

\section{ACKNOWLEDGMENTS}

This project was supported by funding from the National Institutes of Health-National Heart, Lung, and Blood Institute (R34 HL131726). This work is also a publication of the US Department of Agriculture, Agricultural Research Service, Children's Nutrition Research Center, Department of Pediatrics, Baylor College of Medicine, Houston, TX and funded in part with federal funds from the USDA, Agricultural Research Service under cooperative agreement no. 58-3092-5-001.

\section{SUPPLEMENTARY DATA}

Supplementary data related to this article can be found at https://doi. org/10.1016/j.jneb.2020.11.006.

\section{REFERENCES}

1. Wake M, Nicholson JM, Hardy P, Smith K. Preschooler obesity and parenting styles of mothers and fathers: Australian national population study. Pediatrics. 2007;120:e1520-e1527.

2. Morgan PJ, Young MD, Lloyd AB, et al. Involvement of fathers in pediatric obesity treatment and prevention trials: a systematic review. Pediatrics. 2017; 139:e20162635.

3. Fraser J, Skouteris H, McCabe M, Ricciardelli LA, Milgrom J, Baur LA. Paternal influences on children's weight gain: a systematic review. Fathering. 2011;9:252-267

4. Davison KK, Kitos N, Aftosmes-Tobio A, et al. The forgotten parent: fathers' representation in family interventions to prevent childhood obesity. Prev Med. 2018;111:170-176.

5. O'Connor T, Perez O, Garcia IC, Gallagher M. Engaging Latino fathers in children's eating and other obesityrelated behaviors: a review. Curr Nutr Rep. 2018;7:29-38.

6. Freeman E, Fletcher R, Collins CE, Morgan PJ, Burrows T, Callister R.
Preventing and treating childhood obesity: time to target fathers. Int $J$ Obes (Lond). 2012;36:12-15.

7. Brophy S, Rees A, Knox G, Baker JS, Thomas NE. Child fitness and father's BMI are important factors in childhood obesity: a school based crosssectional study. PLoS One. 2012;7: e36597.

8. Aris IM, Bernard JY, Chen LW, et al. Modifiable risk factors in the first 1000 days for subsequent risk of childhood overweight in an Asian cohort: significance of parental overweight status. Int J Obes (Lond). 2018;42:44-51.

9. Morgan PJ, Young MD. The influence of fathers on children's physical activity and dietary behaviors: insights, recommendations and future directions. Curr Obes Rep. 2017;6:324-333.

10. Ogden CL, Carroll MD, Kit BK, Flegal KM. Prevalence of childhood and adult obesity in the United States, 20112012. JAMA. 2014;311:806-814.

11. Hales CM, Carroll MD, Fryar CD, Ogden CL. Prevalence of obesity among adults and youth: United States, 20152016. NCHS Data Brief. 2017:(288):1-8.

12. Portacio FG, Botero P, St George SM, Stoutenberg M. Informing the adaptation and implementation of a lifestyle modification program in Hispanics: a qualitative study among low-income Hispanic adults. Hisp Health Care Int. 2018;16:204-212.

13. Corona E, Flores YN, Arab L. Trends in evidence-based lifestyle interventions directed at obese and overweight adult Latinos in the US: a systematic review of the literature. J Community Health. 2016;41:667-673.

14. Cabrera NJ, Tamis-LeMonda CS, Bradley RH, Hofferth S, Lamb ME. Fatherhood in the twenty-first century. Child Dev. 2000;71:127-136.

15. Morgan PJ, Lubans DR, Callister R, et al. The 'Healthy Dads, Healthy Kids' randomized controlled trial: efficacy of a healthy lifestyle program for overweight fathers and their children. Int J Obes (Lond). 2011;35:436447.

16. Morgan PJ, Collins CE, Plotnikoff RC, et al. The 'Healthy Dads, Healthy Kids' community randomized controlled trial: a community-based healthy lifestyle program for fathers and their children. Prev Med. 2014;61:90-99.

17. Bandura A. Health promotion by social cognitive means. Health Educ Behav. 2004;31:143-164. 
18. Golan M, Weizman A. Familial approach to the treatment of childhood obesity: conceptual mode. J Nutr Educ. 2001;33:102-107.

19. Morgan PJ, Young MD, Smith JJ, Lubans DR. Targeted health behavior interventions promoting physical activity: a conceptual model. Exerc Sport Sci Rev. 2016;44:71-80.

20. Morgan PJ, Lubans DR, Plotnikoff RC, et al. The 'Healthy Dads, Healthy Kids' community effectiveness trial: study protocol of a community-based healthy lifestyle program for fathers and their children. BMC Public Health. 2011;11:876.

21. Morgan PJ, Collins CE, Lubans DR, et al. Twelve-month outcomes of a father-child lifestyle intervention delivered by trained local facilitators in underserved communities: the Healthy Dads Healthy Kids dissemination trial Transl Behav Med. 2019;9:560-569.

22. O'Connor TM, Perez O, Beltran A, et al. Cultural adaptation of 'Healthy Dads, Healthy Kids' for Hispanic families: applying the Ecological Validity Model. Int J Behav Nutr Phys Act. 2020;17:52.

23. Marin G, Marin BV. Research With Hispanic Populations. Newbury
Park, CA: Sage Publications, Inc; 1991.

24. US Department of Health and Human Services. Physical activity guidelines for Americans. https://health.gov/dietaryguidelines/2015/guidelines/appendix1/. Accessed October 22, 2020.

25. US Department of Agriculture. ChooseMyPlate. https://www.choosemyplate. gov/. Accessed October 22, 2020.

26. Reid Chassiakos YL, Radesky J, Christakis D, Moreno MA, Cross C. Council on Communications and Media. Children and adolescents and digital media. Pediatrics. 2016;138:e20162593.

27. O'Connor TM, Beltran A, Musaad S, et al. Feasibility of targeting Hispanic fathers and children in an obesity intervention: Papás Saludables Niños Saludables. Child Obes. 2020;16:379-392.

28. Feldstein AC, Glasgow RE. A Practical, Robust Implementation and Sustainability Model (PRISM) for integrating research findings into practice. Jt Comm J Qual Patient Saf. 2008;34:228-243.

29. McCreight MS, Rabin BA, Glasgow RE, et al. Using the Practical, Robust Implementation and Sustainability Model (PRISM) to qualitatively assess multilevel contextual factors to help plan, implement, evaluate, and disseminate health services programs. Transl Behav Med. 2019;9:1002-1011.

30. Riebe D, Franklin BA, Thompson PD, et al. Updating ACSM's recommendations for exercise preparticipation health screening. Med Sci Sports Exerc. 2015;47: 2473-2479.

31. Martinez J, Powell J, Agne A, Scarinci I, Cherrington A. A focus group study of Mexican immigrant men's perceptions of weight and lifestyle. Public Health Nurs. 2012;29:490-498.

32. Valdez LA, Morrill KE, Griffith DM, Lindberg NM, Hooker SP, Garcia DO. Mexican origin Hispanic men's perspectives of physical activity-related health behaviors. Am J Mens Health. 2019;13:1557988319834112.

33. Coleman KJ, Ocana LL, Walker C, et al. Outcomes from a culturally tailored diabetes prevention program in Hispanic families from a low-income school: Horton Hawks Stay Healthy (HHSH). Diabetes Educ. 2010;36:784-792.

34. Noe-Bustamante L. Key facts about U.S. Hispanics and their diverse heritage. https://www.pewresearch.org/facttank/2019/09/16/key-facts-about-u-shispanics/. Accessed October 22, 2020.

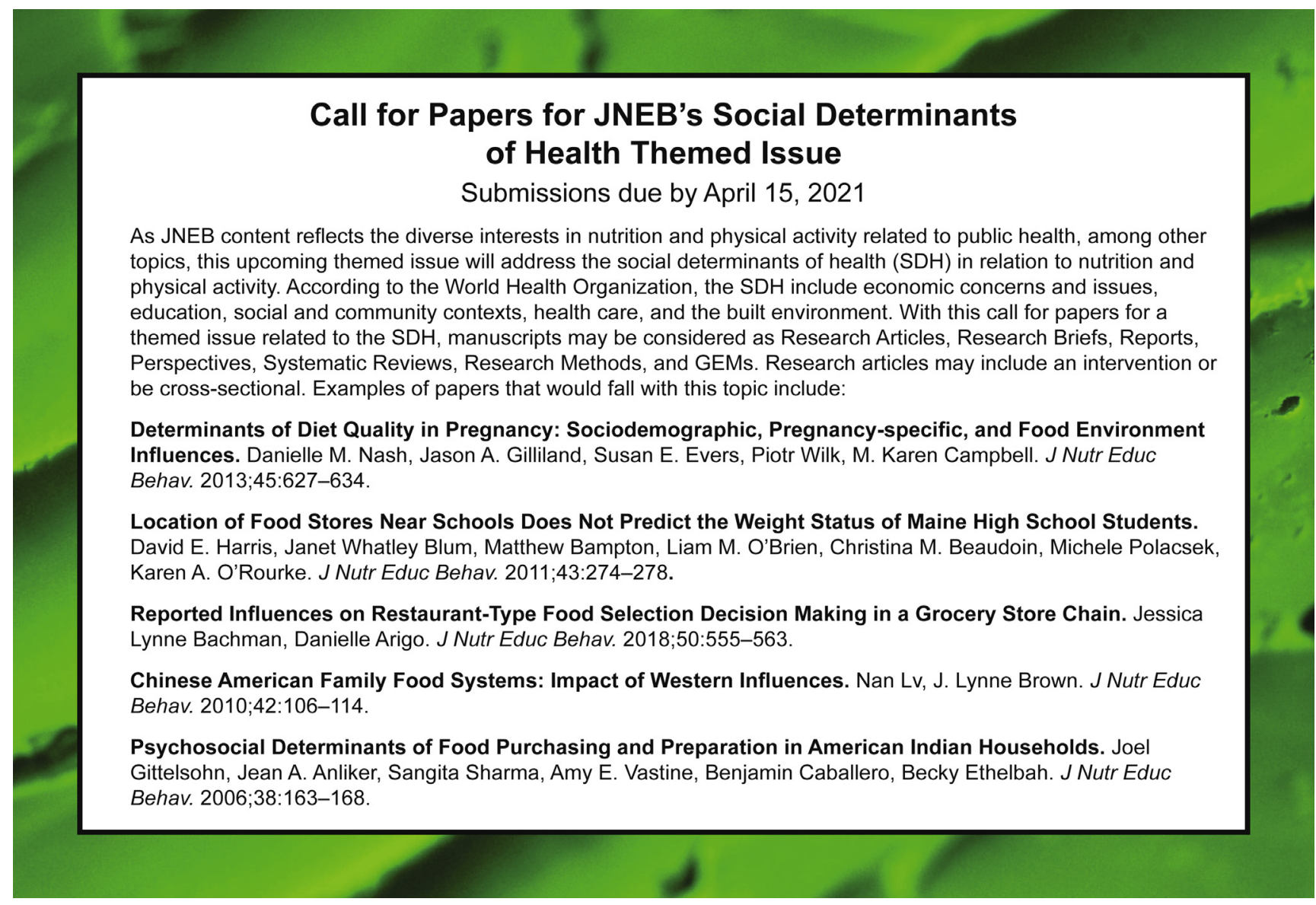

